\title{
The Synthesis of Sterically Hindered Amides
}

\author{
Gabriel Schäfer§ and Jeffrey W. Bode* \\ §SCS-Metrohm Foundation Award for best oral presentation
}

\begin{abstract}
Amide bond formation is one of the most important reactions due to the ubiquity of the amide functional group in pharmaceuticals and biologically active compounds. However, even the best existing methods reach their limits when it comes to the synthesis of sterically hindered amides. In this article we summarize our research in the formation of sterically hindered amides. We show that the direct coupling of Grignard reagents to isocyanates provides a facile and robust solution to this long-standing challenge and hope that this methodology will find widespread application in the synthesis of pharmaceuticals and materials.
\end{abstract}

Keywords: Amide synthesis · $N$-Carboxyanhydrides · Grignard reagents · Isocyanates · Steric hindrance

\section{Introduction}

The synthesis of amide bonds is an integral part of every organic chemistry laboratory and is commonly regarded as the most used chemical reaction in drug discovery. ${ }^{[1]}$ The conventional method to prepare amides is condensation of amines and carboxylic acids in the presence of a coupling reagent (Scheme 1). [2]

The role of the coupling reagent is to transform the carboxylic acid moiety into an activated carboxylate, which is attacked by the amine to generate a new amide bond. Many different coupling reagents are now commercially available, allowing the customer to choose the most suitable reagent for a specific chemical transformation. However, one of the biggest challenges in amide bond formation chemistry cannot be solved even with the best coupling reagent in hand: the synthesis of sterically hindered amides. ${ }^{[3]}$ The difficulties associated with the formation of this class of amides stems from the slow nucleophilic attack of the amine onto the activated carboxylate in a sterically congested environment.

During the last 10 years our group has reported several new amide bond formation methods. ${ }^{[4-7]}$ In 2006, we de- scribed the chemoselective, amide-bond forming ligation of hydroxylamines with $\alpha$-ketoacids (KAHA ligation). ${ }^{[4]}$ This reaction proceeds under mild conditions, requires no reagents or catalysts and only produces water and $\mathrm{CO}_{2}$ as by-products. Although the KAHA ligation can be used to form simple amide products, its major application lies in the synthesis of larger peptides using a C-terminal peptide $\alpha$-ketoacid and an N-terminal hydroxylamine (Scheme 2a). ${ }^{[8]}$ Despite the elegance of this approach, sterically hindered amides are difficult to form via KAHA ligation. In 2012, our group disclosed a new

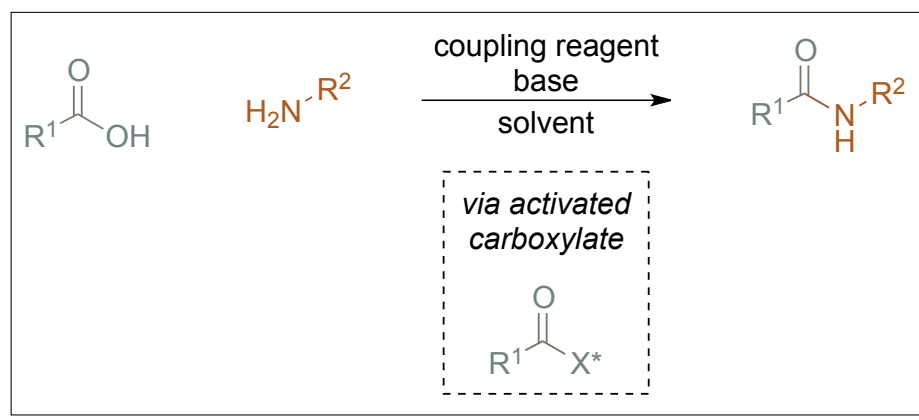

\section{a) KAHA ligation}<smiles>[R]C(=O)C(=O)O</smiles><smiles>[R2]NOC(=O)OCCCC(=O)O[R6]</smiles><smiles>[R]NC([R])=O</smiles>

Scheme 2.

Chemoselective amide-forming ligations by Bode and co-workers. ${ }^{[4-7]}$
Scheme 1. Traditional amide bond formation.
${ }^{*}$ Correspondence: Prof. Dr. J. W. Bode ETH Zürich

Laboratorium für Organische Chemie

Vladimir-Prelog-Weg 1-5

$\mathrm{CH}-8093$ Zürich

Tel.: +41446332103

E-mail: bode@org.chem.ethz.ch b) Acyltrifluoroborate $O$-benzoyl hydroxylamine ligation<smiles>[R2]NO[R6]</smiles> 
amide-forming ligation using acyltrifluoroborates and $O$-benzoyl hydroxylamines, without the need for additional reagents or catalysts (Scheme 2b). ${ }^{[7]}$ This reaction operates under aqueous conditions and gives remarkably rapid amide formation. Many different amides could be prepared in high yields, however highly sterically hindered amides could not be formed. In order to address the challenge of preparing sterically hindered amides, we sought out to find a facile and reliable solution of this longstanding unmet synthetic need.

\section{Addition of Grignard Reagents to Isocyanates}

In our effort to find a practical method for the preparation of sterically hindered amides we reexamined two reports from Gilman on the addition of Grignard reagents to isocyanates to form amide bonds (Scheme 3).[9] Two factors were intriguing to us: i) Gilman described a $\mathrm{C}-\mathrm{C}$ bond forming approach for amide synthesis, which is in contrast to the standard coupling-reagent based $\mathrm{C}-\mathrm{N}$ bond formation process; and ii) the synthetic community, including ourselves, has nearly forgotten this elegant approach of Gilman as only a few applications of this reaction have appeared in the literature in the last 85 years - none of them targeted sterically hindered amides. ${ }^{[10]}$ We reasoned that this was due to the fact that Gilman and coworkers themselves were not interested in the amide products as such, but rather in finding a way to accurately identify and quantify their Grignard reagents.

We were eager to see if the addition of Grignard reagents to isocyanates would allow the synthesis of sterically hindered amides. We began our investigation by using the sterically hindered isocyanate $\mathbf{3}$ as a model substrate. Several bulky Grignard reagents could be added with ease to isocyanate $\mathbf{3}$ and provided the corresponding products in high yields (Scheme 4).[11] The reaction proved to be simple and user-friendly: A solution of the Grignard reagent was added to a solution of isocyanate in ethereal solvent at $0{ }^{\circ} \mathrm{C}$, followed by warming to room temperature. The analytically pure amide was obtained after aqueous workup and washing of the crude material with hexanes.

To explore the versatility of our methodology, we examined several other isocyanates in the reaction with mesitylmagnesium bromide (4, Scheme 5). A wide range of other sterically hindered isocyanates could be successfully used, including tert-butyl, adamantyl, 2,6-dichlorophenyl or 2-fluoro-6-trifluoromethyl isocyanate. Furthermore, sterically hindered $\alpha$-trifluoromethyl-substituted am-

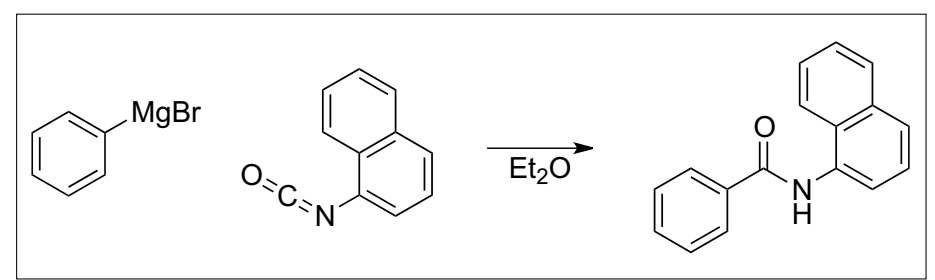

Scheme 3. Identification and quantification of phenylmagnesium bromide via addition to 1-naphthylisocyanate, as described by Gilman et al. ${ }^{[9 b]}$

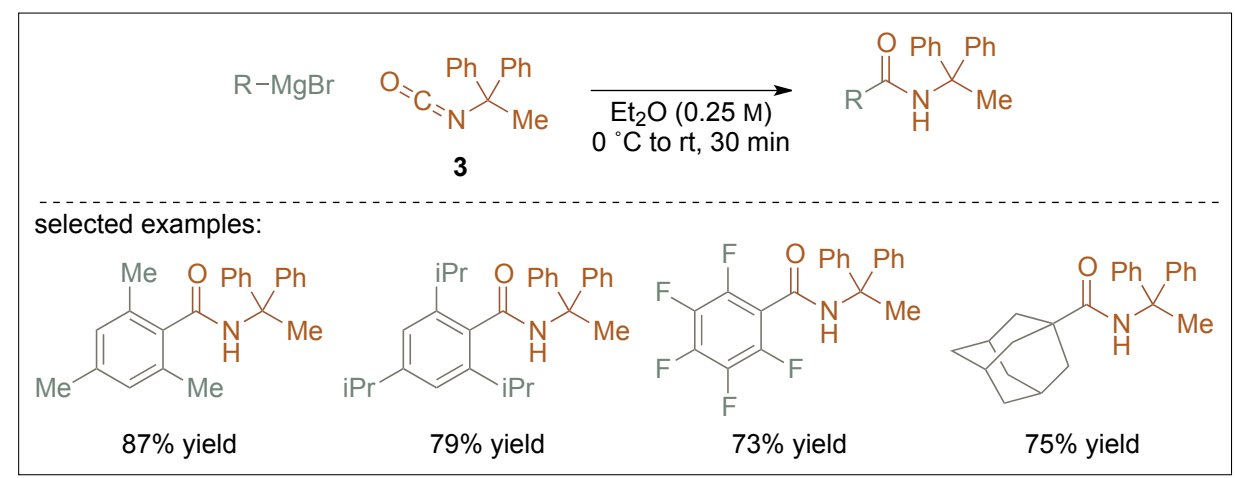

Scheme 4. Addition of sterically hindered Grignard reagents to isocyanate 3.

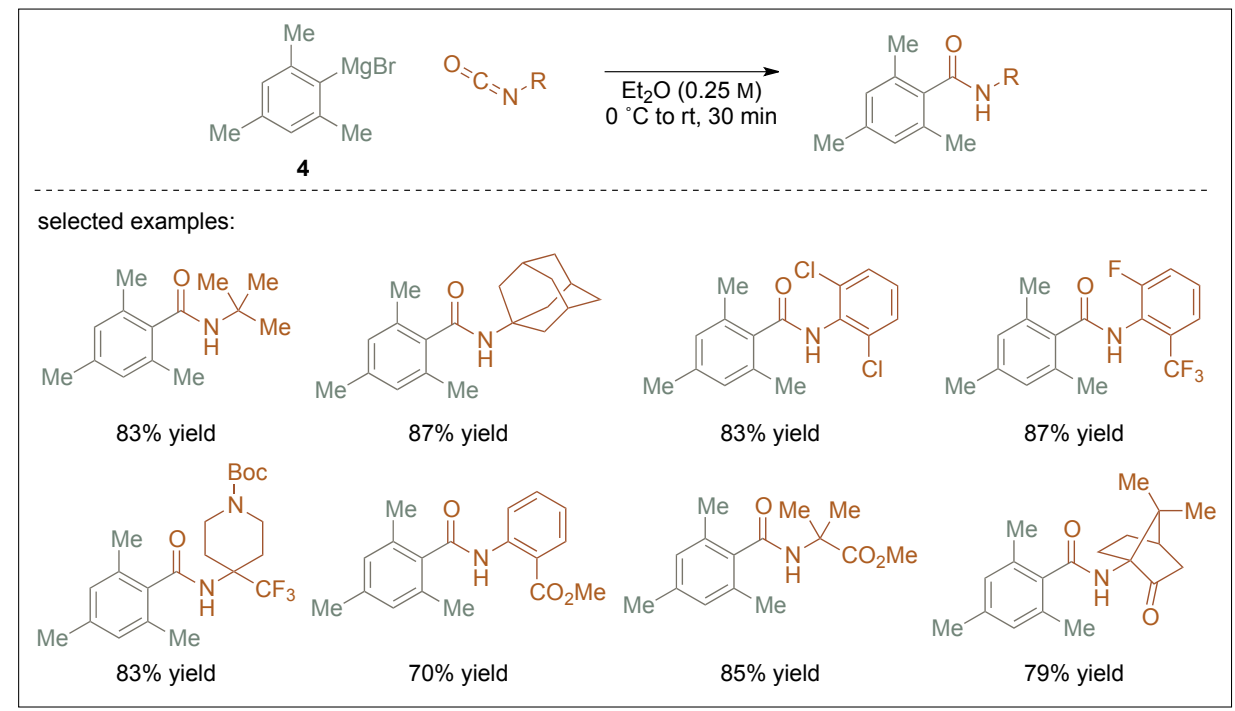

Scheme 5. Addition of mesitylmagnesium bromide (4) to various isocyanates.

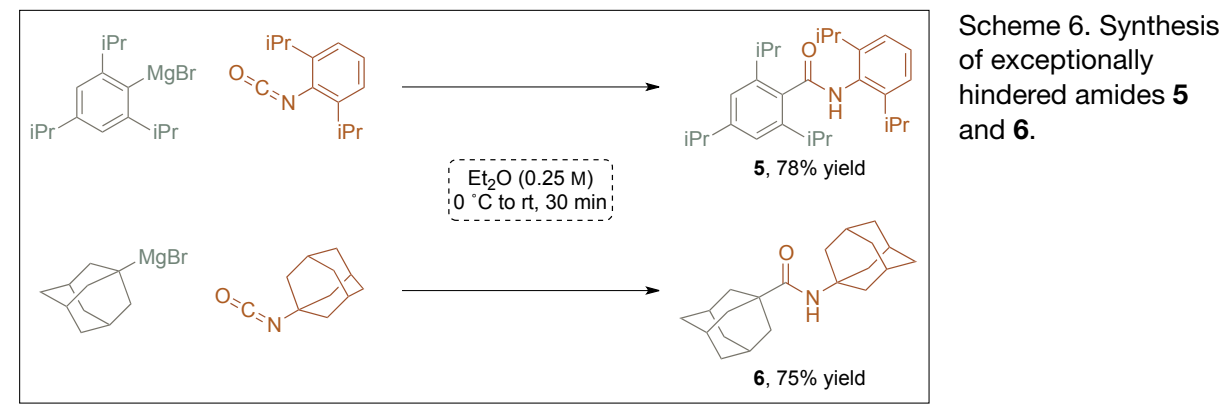

ides could be accessed using our conditions. The chemoselective addition of the Grignard reagent to the isocyanate moiety over ester or ketone functional groups is noteworthy - the corresponding amides were obtained in good yields.

The highlight of our work was the preparation of two extremely hindered amides 5 and $\mathbf{6}$ via the combination of the most hindered substrates from each reaction partner, setting a benchmark in the area of steric hindrance in amide bond synthesis (Scheme 6).

\section{Synthesis of Isocyanates}

A criticism of our work is the use of isocyanates, a class of compounds that is biased with a poor reputation since the 
Bhopal accident with methyl isocyanate. It is important to note that isocyanates with a higher molecular mass, especially sterically hindered examples, are easily handled materials and can be stored over months without any sign of decomposition. Additionally, isocyanates are highly appreciated starting materials in polymer chemistry and many of them are commercially available ${ }^{[12]}$ or can be readily prepared via well-established methods, starting from either carboxylic acids or amines. The sterically hindered isocyanates prepared via these methods can be purified by column chromatography or distillation (Scheme 7). ${ }^{[10]}$

\section{4. $\mathbf{N}$-Carboxyanhydrides as $\alpha$-Isocyanatocarboxylic Acid Surrogates}

Despite the broad substrate scope of the isocyanate-Grignard methodology, one substrate class could not be directly accessed: sterically hindered, $\mathrm{N}$-acylated amino acids. The reason for this was the lack of a suitable starting material, namely $\alpha$-isocyanatocarboxylic acids. We sought to find a solution for this limitation and were intrigued by the extensive literature on $\mathrm{N}$-carboxyanhydrides (NCA) - a substrate class commonly used as starting materials in the preparation of polypeptides via ring-opening polymerization.[13]

We speculated that NCAs could serve as $\alpha$-isocyanatocarboxylic acid surrogates in combination with two equivalents of a Grignard reagent. The first equivalent of Grignard reagent serves as a base and deprotonates the $\mathrm{N}-\mathrm{H}$ proton, upon which an $\alpha$-isocyanatocarboxylate is released. This reactive intermediate is subsequently attacked by the second equivalent of organometallic reagent to form the desired amide bond (Scheme 8 ).

When 3-oxa-1-azaspiro[4.5]decane2,4-dione (7) was treated with mesitylmagnesium bromide (4) at $-78{ }^{\circ} \mathrm{C}$ and subsequently warmed to room temperature, the corresponding gem-disubstituted, $\mathrm{N}$-acyl amino acid was isolated in nearly quantitative yield (Scheme 9). ${ }^{[14]}$

The substrate scope of the Grignard reagent and the NCA was found to be very broad and several highly sterically hindered, $N$-acylated amino acids could be obtained in good to high yield, including products with sensitive functional groups (Scheme 10). Organolithium reagents were also viable coupling partners in this reaction and several interesting heterocyclic products could be obtained (Scheme 11).

The existence of an $\alpha$-isocyanatocarboxylate intermediate was successfully established using in situ IR spectroscopy: When two equivalents of mesitylmagne- a) From carboxylic acids<smiles>CC(C(=O)O)(c1ccccc1)c1ccccc1</smiles><smiles>[N-]=[N+]=NP(=O)(Oc1ccccc1)Oc1ccccc1</smiles>

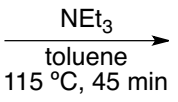

$\mathrm{O}_{\mathrm{C}_{\mathrm{N}}} \mathrm{X}_{\mathrm{Me}}^{\mathrm{Ph}}$

3, $85 \%$ yield

b) From amines<smiles>CCCCCCCCCCCCC(=O)N1CCC(C)(C(=O)OCc2ccccc2)CC1</smiles>
$\mathrm{HCl} \cdot \mathrm{H}_{2} \mathrm{~N}>\mathrm{CF}_{3}$<smiles>O=C(OC(Cl)(Cl)Cl)OC(Cl)(Cl)Cl</smiles>
sat. aq. $\mathrm{NaHCO}_{3} /$ triphosgene $\mathrm{CH}_{2} \mathrm{Cl}_{2}(1: 1)$
$0^{\circ} \mathrm{C}, 30 \mathrm{~min}$<smiles>CC(C)(C)OC(=O)N1CCC(N=C=O)(C(F)(F)F)CC1</smiles>
$65 \%$ yield
Scheme 7. Synthesis of isocyanates.
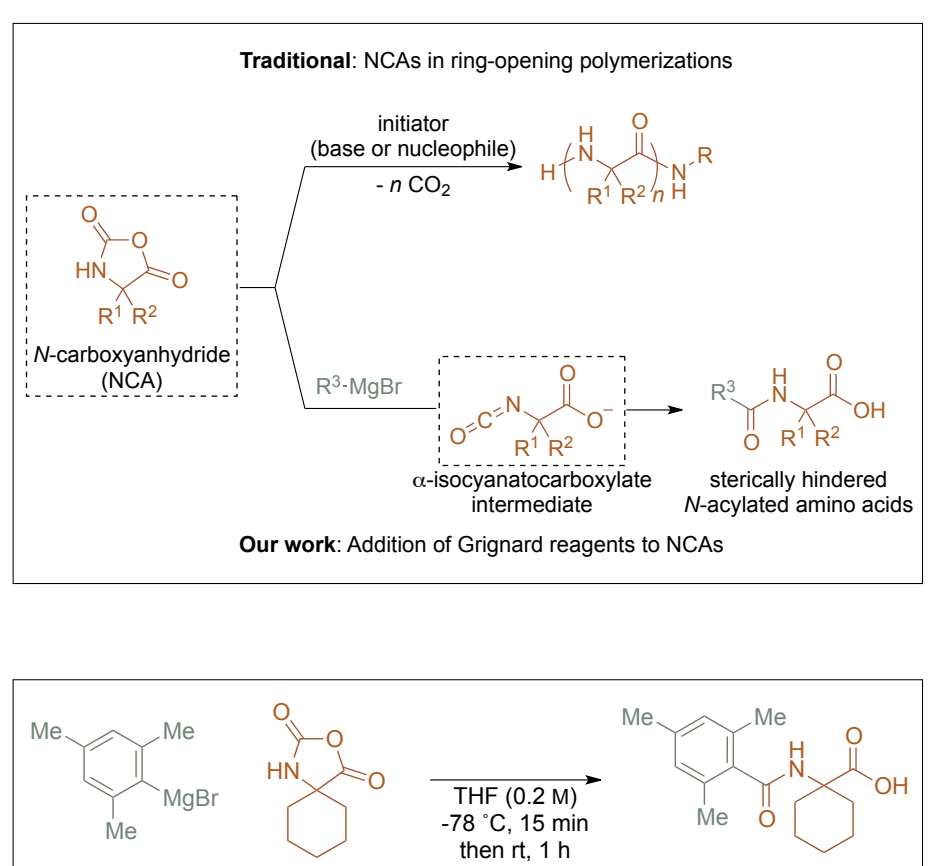

4 (2.1 equiv)

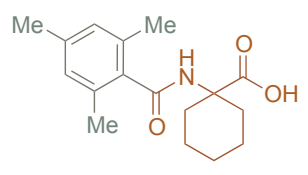

$93 \%$ yield
Scheme 8.

$\mathrm{N}$-Carboxyanhydrides (NCAs) in polymer chemistry and in the synthesis of sterically hindered, $N$-acylated amino acids.
Scheme 9. Addition of mesitylmagnesium bromide (4) to NCA 7

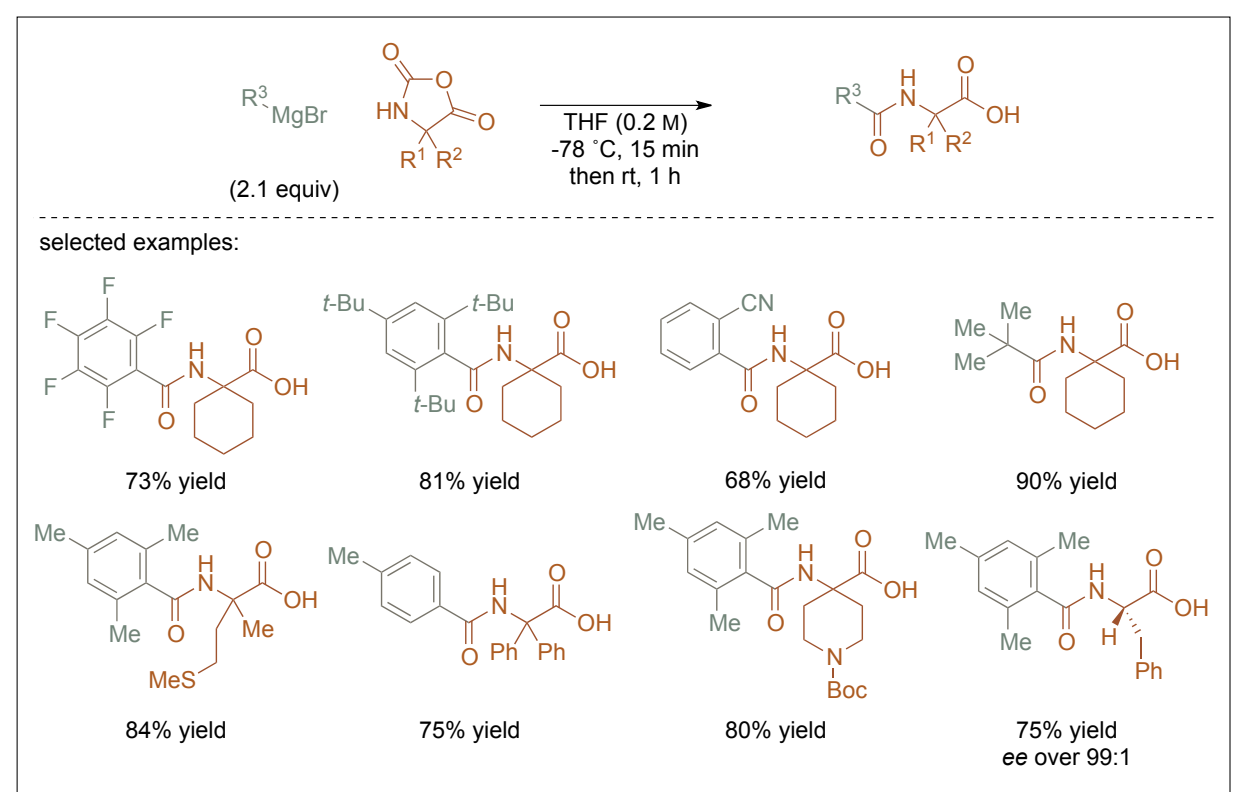

Scheme 10. Addition of Grignard reagents to $N$-carboxyanhydrides. 


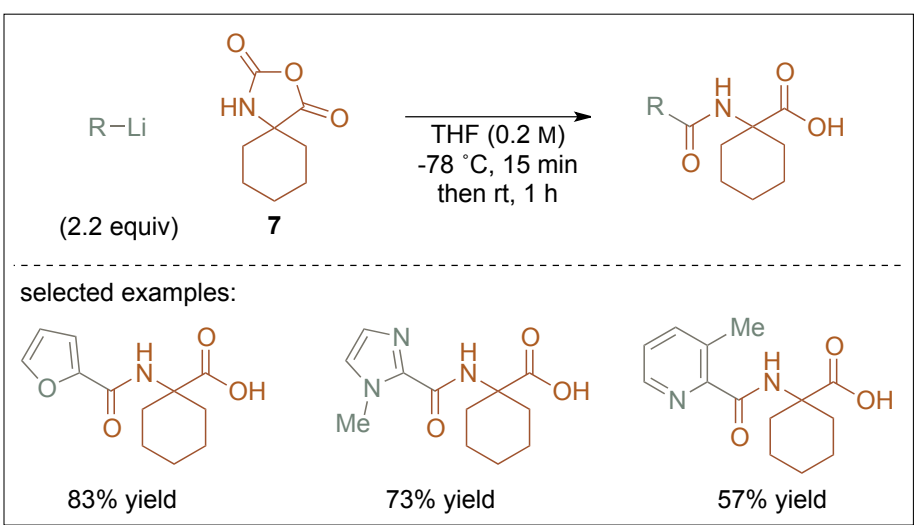

sium bromide (4) were added to a solution of 3-oxa-1-azaspiro[4.5]decane-2,4-dione (7) an intense absorption at $2250 \mathrm{~cm}^{-1}$ appeared immediately, consistent with the typical valence vibration of isocyanates.

\section{Conclusions}

The synthesis of sterically hindered amides is considered as an unsolved challenge in the area of amide bond formation. The addition of Grignard reagents to isocyanates provides a facile and robust solution to this problem and a wide range of sterically hindered amides can be synthesized with ease and in high yields. ${ }^{[10]}$ Most of the described amide products in this report would be difficult to synthesize
Scheme 11. Addition of organolithium reagents to NCA 7.
[1] S. D. Roughley, A. M. Jordan, J. Med. Chem. 2011, 54, 3451.

[2] E. Valeur, M. Bradley, Chem. Soc. Rev. 2009, $38,606$.

[3] V. R. Pattabiraman, J. W. Bode, Nature 2011, 480, 471.

[4] J. W. Bode, R. M. Fox, K. D. Baucom, Angew. Chem. Int. Ed. 2006, 45, 1248 .

[5] J. W. Bode, S. S. Sohn, J. Am. Chem. Soc. 2007, 129, 13798.

[6] V. R. Pattabiraman, A. O. Ogunkoya, J. W Bode, Angew. Chem. Int. Ed. 2012, 51, 5114.

[7] A. M. Dumas, G. A. Molander, J. W. Bode, Angew. Chem. Int. Ed. 2012, 51, 5683.

[8] a) T. Fukuzumi, L. Ju, J. W. Bode, Org. Biomol. Chem. 2012, 10, 5837; b) Y.-L. Huang, R. Frey, M. E. Juarez-Garcia, J. W. Bode, Heterocycles 2012, 84, 1179; c) J. Wu, J. Ruiz-Rodríguez, J. M. Comstock, J. Z. Dong, J. W. Bode, Chem. Sci. 2011, 2, 1976.

[9] a) H. Gilman, C. R. Kinney, J. Am. Chem. Soc. 1924, 46, 493; b) H. Gilman, M. Furry, J. Am. Chem. Soc. 1928, 50, 1214.

proaches. The Grignard-isocyanate protocol does not need an excess amount of reagents, the reactions are complete within minutes at room temperature and the products are easily purified. Furthermore, the addition of organometallic species to NCAs opens up a new way for the synthesis of sterically hindered, $N$-acylated amino acids. The reaction proceeds via an $\alpha$-isocyanatocarboxylate intermediate, whose existence was demonstrated by react-IR. ${ }^{[13]}$ We hope that the direct coupling of isocyanates with Grignard reagents will find a widespread use in the preparation of challenging amides, both in academic and industrial laboratories.

Received: January 30, 2014

[10] a) H. M. Singleton, W. R. Edwards, Jr., J. Am. Soc. Chem. 1938, 60, 540; b) L. Field, J. E. Lawson, J. W. McFarland, J. Am. Chem. Soc. 1956, 78, 4389; c) J. W. McFarland, R. L. Harris, J. Org. Chem. 1967, 32, 1273; d) K. A. Parker, E. G. Gibbons, Tetrahedron Lett. 1975, 16, 981; e) Y. Zhang, J. Jiang, Y. Chen, Tetrahedron Lett. 1987, 28, 3815; f) I. Stefanuti, S. A. Smith, R. J. K. Taylor, Tetrahedron Lett. 2000, 41, 3735; g) A. Padwa, K. R. Crawford, P. Rashatasakhon, M. Rose, J. Org. Chem. 2003, 68, 2609; h) M. I. Antczak, J. M. Ready, Chem. Sci. 2012, 3, 1450.

[11] G. Schäfer, C. Matthey, J. W. Bode, Angew. Chem. Int. Ed. 2012, 51, 9173.

[12] E. Delebecq, J.-P. Pascault, B. Boutevin, F. Ganachaud. Chem. Rev. 2013, 113, 80.

[13] H. R. Kricheldorf, Angew. Chem. Int. Ed. 2006, 45,5752 .

[14] G. Schäfer, J. W. Bode, Org. Lett. 2014, 16, 1526. 